Sociologie et sociétés

\title{
La sociologie du théâtre et de la littérature d'après Lucien \\ Goldman
}

\author{
The Sociology of Theater and of Literature, According to Lucien \\ Goldmann
}

\section{La sociología del teatro y de la literatura según Luden Goldmann}

\section{Gibert TARRAB}

Volume 3, numéro 1, mai 1971

URI : https://id.erudit.org/iderudit/001787ar

DOI : https://doi.org/10.7202/001787ar

Aller au sommaire du numéro

Éditeur(s)

Les Presses de l'Université de Montréal

ISSN

0038-030X (imprimé)

1492-1375 (numérique)

Découvrir la revue

Citer cet article

TARRAB, G. (1971). La sociologie du théâtre et de la littérature d'après Lucien Goldman. Sociologie et sociétés, 3(1), 15-24. https://doi.org/10.7202/001787ar

\section{Résumé de l'article}

À partir des principales œuvres de Lucien Goldmann, décédé en octobre 1970 à Paris, Gilbert Tarrab tente dans cet article de montrer l'importante contribution de cet auteur prolifique en matière de sociologie de la littérature et du théâtre. Goldmann a en effet introduit le premier les concepts - qu'il a essayé sa vie durant de rendre opératoires - de " catégorie mentale ", de " cohérence interne d'une œuvre ", de " vision du monde ", de " structure génétique ", notamment. Tous ses écrits tentent en vérité de trouver la structure englobante (sociologique et historique à la fois) dans laquelle seraient insérées les structures internes de l'œuvre étudiée, dans une époque sociale déterminée. Ses analyses procèdent de deux mouvements primordiaux : comprendre l'œuvre (examiner ses structures internes) et l'expliquer (comment l'œuvre s'insère dans la structure socio-économique globalisante). C'est cette dialectique et cette logique que l'auteur de cet article a voulu dégager avec le plus de clarté possible. 


\section{La sociologie du théâtre et de la littérature d'après Lucien Goldmann}

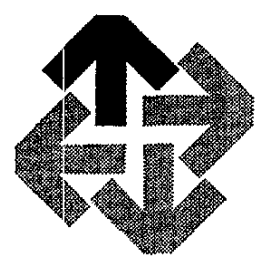

GILBERT TARRAB

LuCIen Goldmann a LAISSÉ à la sociologie de la connaissance - et à la sociologie de la littérature et du théâtre en particulier - un champ de recherches et des études pénétrantes que nous essayerons de circonscrire le plus fidèlement possible dans le présent article.

S'inspirant des travaux de Georg Lukács, Goldmann est surtout l'artisan d'une méthode d'analyse nouvelle des textes littéraires et théâtraux, et originale à bien des égards. On sait en effet qu'il a développé assez tôt une méthodologie épistémologique et positive qu'il a appelée «structuralisme génétique ».

L'idée centrale de la théorie goldmannienne réside en ceci : les structures prégnantes d'une cuvre prégnante - quelle qu'elle soit : la seule condition consiste en sa significativité et sa congruence - ne sont en fait que le reflet des structures mentales des groupes sociaux qui, objectivement, ont élaboré l'univers syntaxique et donc idéologique de l'œuvre en question.

Cependant, il faut ici, d'ores et déjà, se méfier d'une tentation d'extrapolation facile : Goldmann, en effet, s'est toujours considéré comme étant aux antipodes de la doctrine formaliste russe des années 1920, qui est toutefois à l'origine du mouvement structuraliste contemporain, développé notamment par Michel Fou- 
cault pour la philosophie ${ }^{1}$, par Claude Lévi-Strauss pour l'anthropologie culturel$l^{2}$, par Louis Althusser pour l'économie politique ${ }^{3}$.

Contrairement aux prémisses et fondements épistémologiques de l'école structuraliste «pure», Lucien Goldmann propose une approche des textes littéraires et théâtraux plus «positive », plus ancrée dans l'histoire réelle et concrète des hommes : «Des travaux récents, qui essaient de retrouver l'histoire et soulignent à juste titre le caractère a-historique du structuralisme non génétique et du fonctionnalisme, mais qui ne les mettent pas en question et reconnaissent leur validité dans l'étude des expressions symboliques et des relations sociales pour situer l'histoire dans une action abstraite et complètement étrangère à la vie réelle des hommes, nous paraissent, en dernière instance, tout aussi a-historiques. C'est ainsi qu'à un moment où tout ce qui est vraiment important sur le plan de la création littéraire et artistique, depuis le nouveau roman jusqu'aux films de Godard, RobbeGrillet, Visconti, Antonioni et Resnais, est centré sur le caractère inhumain et aculturel du capitalisme d'organisation, sur la difficulté de s'y adapter, la sociologie contemporaine s'y intègre de plus en plus et en devient, sur le plan théorique, un élément constitutif et même souvent, explicitement ou implicitement, son défenseur ${ }^{4}$. Et plus loin : «Il importe [pour le sociologue ] de s'efforcer de connaître la signification des principaux courants de la sociologie contemporaine, la nature et leur lien avec la réalité sociale contemporaine, le sens de leur action à l'intérieur de cette réalité et les cadres sociaux qui pourraient favoriser ou au contraire diminuer leur valeur positive en tant qu'instruments de connaissance ${ }^{5}$.»

Dès lors, on voit combien Goldmann sera intéressé à expliquer et à comprendre les ceuvres romanesques (Robbe-Grillet, Nathalie Sarraute), théâtrales (Racine, Genet) et philosophiques (Kant, Pascal), non pas à partir de la biographie des créateurs, mais à partir d'une analyse serrée de la vie socio-économique réelle dans laquelle ces auteurs ont imaginé leurs écrits, à des niveaux différents. Qui plus est : il essayera de montrer, dans son célèbre Dieu caché ${ }^{6}$ par exemple, comment sont en fait interreliées les œuvres philosophiques de Pascal et de Kant, et l'œuvre dramatique de Racine, dans une trame et une «vision du monde » fondamentalement jansénistes.

En d'autres termes encore, l'approche que Goldmann privilégie est donc la suivante : dans quelle mesure la structure de l'œuvre entière d'un romancier ou d'un dramaturge cohérent avec lui-même et congruent avec son époque, dans sa conception et sa «vision» du monde, dans son écriture scénique ou littéraire (au sens de « littéralité »), dans son langage enfin, peut être révélatrice du même coup et dans le même mouvement - en sous-jacent — de la structure socio-économicopolitique de la société dans laquelle il a été amené à écrire ce qu'il a écrit.

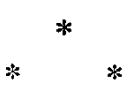


Revenons à la notion de «structuralisme». Il nous faut rappeler ici ce que Roland Barthes écrivait dans Critique et Vérité : "Voilà cent ans que l'on discute autour du mot «structure »; il y a plusieurs structuralismes : génétique, phénoménologique, etc. ; il y a aussi un structuralisme "scolaire», qui consiste à donner le «plan » d'une cuvre. De quel structuralisme s'agit-il [...] [quand on parle] de la «structure » du roman [ou d'une pièce de théâtre] ? [...] Toute l'objectivité du critique tiendra donc, non au choix $d u$ code, mais à la rigueur avec laquelle il appiliquera à l'auvre le modèle qu'il aura choisi ${ }^{7}$. »

Lucien Goldmann a choisi un modèle structuraliste résolument génétique dans sa portée : ce n'est d'ailleurs pas un hasard si le Dieu caché est dédié à Jean Piaget. Et le moins que l'on puisse dire au sujet de ses analyses et nombreuses études consacrées à la sociologie de la littérature et du théâtre, c'est qu'elles sont toutes soustenclues d'une pensée rigoureusement cohérente, quant au code de décryptage pour lequel il a opté. Nous allons tenter d'exposer ce code le plus clairement possible, mais il est évident que le cadre imposé par un article nous oblige à être succinct.

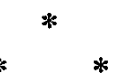

Alberto Moravia a très bien vulgarisé l'idée-force de la théorie goldmannienne : «Lies partisans du Nouveau Roman utilisent les thèses de Goldmann : la société capitaliste est aliénée et corrompue. En disloquant les formes romanesques, le nouveau roman prétend faire, sur le plan littéraire, ce que fait la société capitaliste sur le plan du réel, et donc dénoncer celle-ci ${ }^{8}$.

Cette transposition confirme, par ailleurs, la théorie du fétichisme de la marchandise que Goldmann appelle, à l'instar de Lukács (sur lequel, comme à son habitude, Goldmann insiste beaucoup, en particulier sa «théorie du roman ») : la réification. La grande idée de Lukács est que l'écrivain peut créer un univers complètement imaginaire, mais les joies du héros reflètent les joies de la société d'où l'éctivain est issu. Dans Pour une sociologie du roman ${ }^{9}$, Goldmann s'essaye à donner une définition opérationnelle de sa «méthode génétique-structuraliste », adaptée cette fois (après le Dieu caché, où il donnait une vision tragique des Penisées de Pascal, et du théâtre de Racine, Phèdre en particulier) à l'histoire de la littérature.

L'un des tous premiers problèmes est «celui de la relation entre la FORME ROMANESQUE elle-même et la STRUCTURE du milieu social à l'intérieur duquel elle s'est développée, c'est-à-dire du roman comme genre littéraire et de la société individualiste moderne» (p. 35). Cette forme romanesque, et c'est là l'idée intéressante qu'il apporte, est la transposition sur le plan littéraire de la vie quotidienne darıs la société individualiste née de la production pour le marché. En d'autres termes, le roman est «l'histoire d'une recherche dégradée - que Lukács appelle « démoniaque»-, recherche de valeurs authentiques dans un monde dégradé lui aussi, mais à un niveau autrement avancé et sur un mode différent $\gg$ (p. 23).

D'autre part, les «catégories mentales » qui existent dans un groupe n'apparaissent que sous forme de tendances plus ou moins cohérentes que Goldmann

7. R. Barthes, Critique et Vérité, Paris, Seuil, 1967, p. 19-20.

8. Dans un article du Figaro littéraire du 6 janvier 1966, intitulé : « La mort du roman traclitionnel s.

9. L. Goldmann, Pour une sociologie du roman, Paris, Gallimard, 1964, 372 p. 
appelle «vision du monde». D'où l'idée de «structure»: Goldmann affirme l'existence d'un lien entre les grandes œuvres culturelles et celle des groupes sociaux orientés vers une restructuration globale de la société ou vers sa conservation, l'hypothèse de départ étant qu'on pouvait réunir un certain nombre de faits en une UNITÉ STRUCTURELLE, de telle sorte que, par approximations successives, on arriverait à une « hypothèse structurelle pouvant rendre compte d'un ensemble parfaitement cohérent de faits $\gg$ (p. 351). Le grand intérêt de l'ouvrage réside, en fait, dans l'application des analyses théoriques structurelles et des catégories mentales à l'œuvre de Malraux. Goldmann se base sur le livre de René Girard - Mensonge romantique et vérité romanesque - et lui emprunte l'idée de «médiation»: la recherche de Girard est menée par un héros "problématique », dont l'existence et les valeurs le situent devant des problèmes dont il ne pourrait prendre une conscience claire et rigoureuse : chez Malraux, ce « héros problématique » est bien lié à l'histoire et au développement de la bourgeoisie, mais il n'exprime pas la conscience de cette classe, et n'incarne pas la conscience oppositionnelle du prolétariat.

Goldmann a su dégager, de façon convaincante, les implications politiques de l'œuvre de Malraux, en les rattachant aux tendances dominantes du Parti communiste, et aux fonctions de l'érotisme dans ses romans (opposition de l'érotisme et de l'amour). Enfin, et ce n'est guère l'apport le moins important, l'auteur souligne que la crise de la culture occidentale, à travers les romans de Malraux, a aussi une signification «structurale 》: «Après la disparition des cultures transcendantes du Moyen Âge, [cette crise] résulte de la crise des valeurs individualistes qui, dans la culture classique, avaient remplacé la divinité, et de l'impossibilité de créer des structures ou des formes nouvelles qui ne sauraient plus s'appuyer ni sur le transindividuel ni sur l'individu » (p. 79). Quant à la partie de l'ouvrage consacrée au "Nouveau roman et la réalité », elle est beaucoup moins étoffée, surtout en ce qui concerne l'œuvre de Nathalie Sarraute. L'idée essentielle à retenir est que pour Goldmann - et là, l'analyse structurale ne nous apprend rien de nouveau - la transformation interne du roman traditionnel au nouveau roman porte sur l'unité structurale personnage-objets, modifiée dans le «sens d'une disparition plus ou moins radicale du personnage et d'un renforcement corrélatif non moins considérable de l'autonomie des objets » (p. 288). Donc, suppression de toute importance essentielle de l'individu et de la vie individuelle, à l'intérieur des structures économiques et, à partir de là, dans l'ensemble de la vie sociale. L'univers des objets est ainsi, à son tour, "RÉIFIÉ 》. Et la réalité humaine s'exprime encore dans la structure et les propriétés des objets, tout constitués en univers autonome qu'ils soient.

Les travaux qui nous sont présentés dans le recueil Littérature et Société ${ }^{10}$ (sous la direction de Goldmann) résultent des recherches entreprises depuis quelques années - dans un domaine si peu inventorié en sciences sociales jusqu'ici par ces mêmes chercheurs qui ont ouvert la brèche et défriché un terrain relativement broussailleux sur lequel ils sont encore loin de se mettre d'accord. C'est que la sociologie de la littérature (des textes littéraires) est une science nouvelle, qui ne possède pas encore - fort heureusement d'ailleurs - des outils opératoires très précis, dans la mesure où c'est une science qui se cherche et qui tente d'apporter

10. Littérature et Société. Problèmes de méthodologie en sociologie de la littérature, Bruxelles, Editions de l'Institut de sociologie de l'Université Libre de Bruxelles, 1968, 224 p. 
un éclairage neuf pour une saisie plus «objective» de la réalité littéraire, donc socio-historique. Car, on le sait, pour Goldmann, aucune sociologie (fût-ce de la littérature) ne saurait être positive si elle n'est historique, de même qu'aucune recherche historique ne saurait être scientifique et positive à moins d'être sociologique. Non seulement il n'y a pas de faits sociaux séparés et d'autres faits humains qui seraient historiques, mais il n'y a même pas deux dimensions distinctes de ces faits qui pourraient être rattachées à deux sciences différentes. Aussi, l'exigence d'étudier des faits humains dans leur structure essentielle et dans leur réalité concrète suppose-t-elle une méthode qui soit à la fois « historique » et «sociologique ».

\section{SOCIOLOGIE ET HISTOIRE}

Il ressort de la lecture de cet ouvrage sur les rapports de la littérature et de la société un dénominateur commun à tous les chercheurs : toute tentative de séparer la sociologie de l'histoire ne saurait aboutir qu'à deux sciences qui seraient l'une et l'autre à la fois contestables et abstraites : voilà pourquoi le structuralisme génétique, dont se réclame Goldmann, est avant tout une position rigoureusement «moniste ». Par conséquent, de même que le structuralisme génétique se refuse à toute séparation entre l'histoire et la sociologie, il ne saurait non plus être question d'accepter une séparation radicale entre les lois fondamentales qui régissent le comportement créateur dans le domaine de la culture et celles qui régissent le comportement «quotidien » de tous les hommes dans la vie sociale et économique. En d'autres termes encore, et pour éviter tout malentendu au sujet du concept de «structure » chez Goldmann (comme n'importe quel terme «à la mode », il est dangereux de l'employer sans l'avoir bien défini au départ), cette notion est pour lui un «fait culturel » qui fait partie intégrante de l'ensemble des comportements humains. Les hommes tendent à élaborer des « catégories mentales » qui puissent répondre au plus grand nombre de problèmes qui se posent à eux. Cet ensemble de catếgories mentales constitue une «structure ». Il y a donc l'être qui structure, et l'être qui destructure. Pour restructurer à nouveau, il faut destructurer, dans un univers de «structurations compartimentales». L'homme se définit ainsi, pour Goldmann, comme l'être qui aspire toujours à la structuration et à la « cohérence », la cohérence (d'une œuvre, par exemple) étant un concept clé dans la terminologie goldmannienne. Ce concept de «cohérence» ayant rencontré auprès de certains sociologues et esthéticiens une réticence (on a surtout mis l'accent sur le fait que ce n'était pas un concept opératoire scientifique), nous croyons utile de prériser ici que, pour Goldmann, ce terme ne doit guère être entendu au sens logique et rationaliste, mais au sens « fonctionnel » (Goldmann parle de « fonctionnalité »), c'est-à-dire : la nécessité interne des comportements dans une situation concrète.

Dès lors, le fait de culture serait un domaine privilégié de la cohérence fonctionnelle. Et l'œuvre « cohérente » serait particulièrement caractérisée chez l'écrivain dont l'univers est précisément caractérisé par une cohérence rigoureuse. Pour Goldmann, ce phénomène est un phénomène « historique » et «sociologique » en même temps. Les structures qu'on peut dégager d'une œuvre cohérente doivent toujours être mises en relation avec les structures de la vie économique et sociale où cette cuvre a vu le jour. 
C'est pourquoi Goldmann s'élève - avec raison, semble-t-il - contre toute interprétation des phénomènes littéraires qui ne tienđrait aucun compte de l'impact historique. Après avoir reconnu combien pouvait être séduisante l'interprétation de Roland Barthes, et à quel point il voyait la nécessité et l'urgence d'intégrer cette interprétation à ses propres travaux en la matière, Goldmann s'empresse d'ajouter : "Mais lorsque Barthes s'est orienté vers l'explication des transformations par un mouvement interne de type cyclique ou de n'importe quelle autre nature, et lorsqu'il a vu dans l'histoire seulement un élément perturbateur, propre à troubler des régularités purement immanentes de certains secteurs de la conscience et du comportement, il m'a semblé que je ne me trouvais plus devant une analyse purement scientifique, mais devant l' \& expression idéologique » de tout un ensemble de courants contemporains de pensée qui, correspondant en cela aux «structures technocratiques » de la société occidentale, tendent à éliminer la réalité de l'histoire $\gg$ (p. 219-220) ${ }^{11}$.

Dans Contribution à la sociologie de la connaissance ${ }^{12}$, l'approche qu'il propose pour comprendre l'œuvre théâtrale de Genet (Haute surveillance, les Bonnes, le Balcon, les Nègres et les Paravents) est intéressante à plus d'un égard, en tout cas certainement originale, bien que souffrant par moments de l'absence d'une assise plus scientifique. Goldmann part de l'idée suivante : Genet a dû intégrer dans son œuvre, et sans doute inconsciemment, certaines expériences historiques décisives de la gauche européenne au cours des dernières décennies, de sorte qu'une pièce comme le Balcon, par exemple, apparaît incohérente et ne saurait être réellement comprise dans toute sa richesse sémantique, sans tenir compte de ces expériences historiques, ou de ces occurrences liées à la conjoncture sociopolitique. Mieux : les problèmes historiques seraient devenus l'élément central, à partir duquel l'œuvre devient compréhensible dans son unité structurelle.

Après avoir montré que le monde de Genet en est un où le pouvoir des dominants est inébranlable, où les dominés-esclaves sont animés d'une haine amoureuse pour les dominants, de telle sorte que les dominés finissent par s'identifier aux dominants, tout en réussissant — sur le plan de l'imaginaire - à les vaincre et à les chasser, dans une espèce de rituel poético-onirique et quasi religieux, Goldmann en arrive à la conclusion que "les dominants ne peuvent exister sans les dominés, car l'existence de ces derniers les constitue en tant que dominants, et bien entendu, aussi l'inverse $»$ (p. 122). Situation très beckettienne, il est vrai : pensez à En attendant Godot, à Fin de partie... Mais tandis que chez Beckett la poésie est blanche, on peut montrer combien elle peut être colorée chez Genet, ce qui recouvre, en définitive, un certain « espoir » chez lui, espoir qu'il est bien difficile de retrouver sous l'aridité beckettienne.

Parlant du Balcon, Goldmann affirme que son thème principal est « la prise de conscience de l'importance de l'exécutif dans une société depuis longtemps dominée et dirigée par cet exécutif et par les propriétaires, mais dans laquelle les hommes s'imaginent encore le pouvoir sous les figures traditionnelles et depuis longtemps encore dépassées de l'évêque, du juge et du général » (p. 125). De même,

11. Voir sa conférence sur «Le structuralisme génétique en sociologie de la littérature » (Littérature et Société, p. 195-222).

12. Contribution à la sociologie de la connaissance, ouvrage collectif, Paris, Editions Anthropos, $1968,206 \mathrm{p}$. 
dans les Nègres, comme les révolutionnaires du Balcon, les nègres ne peuvent pas non plus vaincre ceux qui les dominent; et même derrière la scène, dans le combat effectif, ils ne parviennent qu'à détruire l'un d'entre eux. La victoire n'est encore qu'un espoir éloigné dans le temps et dans l'espace. Au sujet des Bonnes, du Balcon et des Nègres, Goldmann se demande s'il s'agit simplement d'une perspective individuelle, propre à l'auteur Jean Genet, ou, « comme dans le cas de la plupart des grandes œuvres littéraires, de la vision du monde d'un groupe social, élaborée de manière partiellement consciente, partiellement non consciente, à un degré de cohérence tel qu'elle offre au poète déjà les catégories structurées qui seront le fondement de sa transposition littéraire » (p. 132). Suit le développement détaillé de cette hypothèse qui, si elle s'avérait juste et fondée, tendrait à montrer que l'analyse structuralo-génétique de la vie culturelle et surtout de l'œuvre littéraire et clramatique, constituerait un moyen d'accès particulièrement important à la compréhension des structures de la conscience et de la pratique des groupes sociaux existants.

Les Paravents, la dernière pièce de Genet, apporte quelque chose de complètement nouveau, aux yeux de Goldmann, par rapport à l'œuvre du dramaturge. Tout d'abord, la possibilité d'une révolte victorieuse en Algérie (on sait que la pièce se déroule en Algérie, pendant la colonisation française), et peut-être aussi darss d'autres pays du monde. Selon notre sociologue, « les trois ordres de la pièce correspondent à trois concepts fondamentaux de la pensée socialiste européenne : la société de classes, basée sur l'oppression, la société née de la victoire de la révolte et du mouvement de libération, qui a supprimé l'oppression mais qui est encore fondée sur la contrainte, et l'image d'avenir d'une société sans classe et sans contrainte qui occupe, dans la pensée socialiste, la place du royaume des cieux dans l'eschatologie chrétienne 》(p. 138). Voilà pourquoi le personnage de Saïd, le s héros » de la pièce, reste en dernière instance, et à travers sa négativité, un personnage éminemment "positif », pour la première fois dans l'ouvre de Genet. Si la thèse de Goldmann est exacte, on pourrait supposer que Saïd, dans la mesure où il est l'émanation d'un groupe social, c'était le Mai 68 français avant la lettre. Mais qu'est devenu le Mai 68 ? Il y a danger considérable de compromission et d'intégration à l'ordre existant, Goldmann le sait : le danger de corruption est lié à toute pratique réformiste, et on ne peut logiquement s'y opposer que par le refus radical du moindre compromis psychique ou intellectuel avec la société technocratique en voile de construction. C'est aussi la thèse de Marcuse, mais le fait que Saïd reste jusqu'à la fin de la pièce debout, plongé dans sa négativité absolue, rien n'ayant pu le briser, est pour le sociologue de la culture d'une importance particulière. Car il faut comprendre la société à partir de la littérature, et non seulement la littérature à partir de la société.

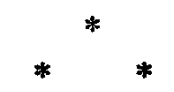

Dans Sociologie de la littérature. Recherches récentes et discussions ${ }^{13}$, Goldmann reprend son travail, déjà paru dans Contribution à la sociologie de la connaissance, sur le théâtre de Genet, mais y ajoute une étude inédite, portant sur les microstructures dans les vingt-cinq premières répliques des Nègres de Jean Genet.

13. Lucien Goldmann et al., Sociologie de la littérature. Recherches récentes et discussions, Bruxelles, Editions de l'Institut de sociologie de l'Université Libre de Bruxelles, * Etudes de sociologie de la littérature $\triangleright, 1970,240 \mathrm{p}$. 
Goldmann et son équipe du Centre de sociologie de l'Université Libre de Bruxelles ont en effet fait le projet d'élaborer un essai de contrôle méthodique d'une de leurs analyses globales concernant le théâtre de Genet, en la confrontant à une de ses pièces, les Nègres, analysée au scalpel, et réplique par réplique : «Le but que nous nous proposions était de recenser les répliques qui s'inséraient difficilement, ou pas du tout, dans notre analyse, ainsi que les éléments et les relations que nous n'avions pas vus et qui présentaient une certaine fréquence dans le texte. Ceci devait nous permettre de modifier éventuellement notre modèle interprétatif de manière à établir son accord avec toutes les répliques de la pièce et de donner une nouvelle dimension à notre analyse en établissant la fréquence et le poids des différents éléments et relations qui constituaient le modèle global » (p. 35).

Cette première recherche formelle, se situant du côté du signifiant cette fois, aussi embryonnaire soit-elle, semble néanmoins répondre à un besoin d'ordre méthodologique. Car Goldmann semble reconnaître enfin, et c'est là, croyons-nous, un grand pas en avant de réalisé, que ses analyses, jusqu'ici toujours limitées au signifié, devaient nécessairement être complétées par des recherches plus strictement formelles (phonétique, stylistique, rythmes et cadences, etc.). Cependant, Goldmann, bien que reconnaissant l'utilité de ces recherches formelles, nuance davantage sa pensée : « Nous affirmons aussi que l'œuvre constitue une unité dans laquelle il est impossible de séparer une forme d'un contenu (ce que les linguistes appellent, dans leur terminologie, signifiant et signifié), et que dans cette unité, la structure significative globale, ayant son origine dans la conscience collective, est nécessairement l'élément premier tant du point de vue chronologique (la conscience collective et les visions du monde existent avant leur expression littéraire) que du point de vue structural, qu'il est par conséquent impossible d'étudier le signifiant avant le signifié [chose qu'il reproche à Lévi-Strauss et à Roman Jakobson], les structures partielles avant la structure de l'Univers, alors que l'inverse est faisable à condition de rester conscient du caractère incomplet et insuffisant de la recherche $\gg$ (p. 37).

\section{CONGLUSION}

Pour Goldmann, il arrive fort souvent que le souci d'unité esthétique de l'écrivain lui fasse écrire une œuvre dont la structure globale, traduite en langage conceptuel par la critique, constitue une vision différente et même opposée à sa pensée, à ses convictions profondes et aux intentions qui l'animaient lorsqu'il a rédigé cette œuvre. Cest pourquoi le sociologue de la littérature - ainsi que le critique, d'ailleurs - doit traiter les « intentions conscientes» de l'auteur comme un «indice» parmi beaucoup d'autres possibles, comme une sorte de réflexion sur l'œuvre, qui lui apporte des suggestions, de même que n'importe quel autre ouvrage critique, mais qu'il doit juger à la lumière du texte, sans lui accorder aucun privilège particulier. Selon Goldmann, « le fait de ne pas surestimer l'importance de l'individu dans l'explication, qui est avant tout la recherche du sujet, individuel ou collectif, pour lequel la structure mentale qui régit l'œuvre a un caractère fonctionnel et significatif ${ }^{14} \%$, est tout à fait essentiel dans la démarche sociologique, quand elle traite des problèmes littéraires, reliés à leur signification socio-historique. Ainsi, il serait

14. L. Goldmann, «Sociologie de la création littéraire ", numéro spécial, de la Revue internationale des sciences sociales, Paris, UNESCO, vol. XIX, no 4, 1968, p. 535. 
impossible d'expliquer la genèse de l'œuvre de Racine et sa signification en la mettant seulement en relation avec la biographie et la psychologie de l'individu Racine : "Le fait que ce qu'on appelle couramment les «influences» n'a aucune valeur explicative et constitue tout au plus une donnée et un problème que le chercheur doit expliquer ${ }^{15}$. »

Bref, la compréhension est un problème de cohérence interne du texte, qui suppose qu'on prenne à la lettre le texte, tout le texte et rien que le texte, et qu'on recherche, à l'intérieur de celui-ci, une structure significative globale.

Dégageant les rapports qu'il peut y avoir entre la sociologie génétique et la psychanalyse, Lucien Goldmann décèle au moins trois éléments communs :

1) L'affirmation que tout comportement humain fait partie d'au moins une structure significative ;

2) Le fait que, pour comprendre ce comportement, il faut l'insérer dans cette structure - que le chercheur se doit de mettre en lumière ;

3) L'affirmation que cette structure n'est réellement compréhensible que si on la saisit dans sa genèse, individuelle ou historique, selon les cas : « Le chercheur arrêtera donc son étude quand il aura mis suffisamment en lumière la relation entre la structure étudiée et la structure englobante pour rendre compte de la genèse de la première en tant que fonction de la seconde ${ }^{16}$.

L'auteur, il est bon de le signaler, a étayé ses positions et argumentations à l'aide d'exemples concrets - qu'il ne nous est pas possible de commenter ici, faute de place - empruntés à ses ouvrages antérieurs, notamment le Dieu caché, Recherches dialectiques et "Le théâtre de Genet : essai d'étude sociologique ${ }^{17}$ », en insistant tout particulièrement sur le théâtre de Racine et de Molière, comme créations collectives et mettant en scène des groupes sociaux réels.

Nous savons combien Goldmann est «contesté » aujourd'hui, par l'intelligentsia bien-pensante, fût-elle du Québec. Il est cependant bon de le relire, aujourd'hui qu'il n'est plus, pour savoir si le temps est venu de le «brûler », comme on a tenté, il y a quelques années, de «brûler» Roland Barthes ${ }^{18}$.

RÉSUMÉ

À partir des principales œuvres de Lucien Goldmann, décédé en octobre 1970 à Paris, Gilbert Tarrab tente dans cet article de montrer l'importante contribution de cet auteur prolifique en matière de sociologie de la littérature et du théâtre. Goldmann a en effet introduit le premier les concepts - qu'il a essayé sa vie durant de rendre opératoires - de "catégorie mentale ", de "cohérence interne d'une cuvre", de "vision du monde ", de "structure génétique ", notamment. Tous ses écrits tentent en vérité de trouver la structure englobante (sociologique et historique à la fois) dans laquelle seraient insérées les structures internes de l'œuvre étudiée, dans une époque sociale déterminée. Ses analyses procèdent de deux mouvements primordiaux :

15. L. Goldmann, "Sociologie de la création littéraire », numéro spécial de la Revue internationale des sciences sociales, Paris, UNESCO, vol. XIX, no 4, 1968, p. 536.

16. Ibid., p. 539.

17. Dans Cahiers Madeleine Renaud-Jean-Louis Barrault, novembre 1966.

18. Signalons qu'un ouvrage posthume de Goldmann, Structures mentales et création culturelle, est actuellement sous presse aux Editions Anthropos, Paris. Le lecteur pourra d'ores et déjà en lire un chapitre : "Problèmes philosophiques et politiques dans le théâtre de JeanPaul Sartre », dans l'Homme et la Société, Paris, Editions Anthropos, $\mathrm{n}^{\circ} 17$, juillet-septembre 1970 , p. 5-34. 
comprendre l'œuvre (examiner ses structures internes) et l'expliquer (comment l'œuvre s'insère dans la structure socio-économique globalisante). C'est cette dialectique et cette logique que l'auteur de cet article a voulu dégager avec le plus de clarté possible.

\section{ABSTRACT}

[The Sociology of Theater and of Literature, According to Lucien Goldmann] Lucien Goldmann died in October 1970 in Paris. Considering his main writings, Gilbert Tarrab tries in this article to show the important contribution of this prolific author to the sociology of literature and of theater. Goldmann indeed was the first to introduce the concepts of " mental category *, * internal coherence of a work», "world view », and c genetic structure » - concepts which he tried to operationalize during his life. In effect, all his writings attempt to find the encompassing structure (both historical and sociological) in which the internal structure of the studied work seems incorporated during a specific social epoque. His analyses proceed from two primordial movements : understand the work (examine its internal structures) and explain it (show how the work is incorporated in the encompassing socio-economic structure). The author of this article wanted to show this dialectic and this logic with the greatest clarity possible.

\section{RESUMEN}

[La sociología del teatro y de la literatura segun Lucien Goldmann] A partir de las principales obras de Lucien Goldmann, fallecido en octubre de 1970 en Paris, Gilbert Tarrab intenta mostrar la importante contribución de este autor fecundo en materia de sociología de la literatura y del teatro. En efecto, Goldmann ha sido el primero en introducir los conceptos de * categoria mental », de "coherencia interna de una obra », de "visión del mundo", de " estructura genética - conceptos que ha tratado, durante toda su obra, de darles un alcance operatorio. Todos sus escritos buscan poder al descubierto la estructura englobante (al mismo tiempo sociológica e histórica) en la cual estarian insertas las estructuras internas de la obra estudeada, en una época social determinada. Sus análisis se efectuan en dos movimientos principales : comprender la obra (examinar sus estructuras internas) y explicarla (mostrar cómo la obra se inserta en la estructura socio-económica globalizante). Es esta dialectica y esta lógica lo que el autor de este artículo ha tratado de explicitar con la mayor claridad posible. 